\title{
ACUTE AND CHRONIC MORBIDITY DIFFERENCES BETWEEN MUSCLE-SPARING AND STANDARD LATERAL THORACOTOMIES
}

Rodney J. Landreneau, MD Frank Pigula, $\mathrm{MD}^{\mathrm{b}}$

James D. Luketich, $\mathrm{MD}^{\mathrm{b}}$

Robert J. Keenan, MD $^{\mathrm{b}}$

Susan Bartley, RN ${ }^{\mathrm{b}}$

Lynda S. Fetterman, $\mathrm{RN}^{\mathrm{a}}$

Claudia M. Bowers, RN ${ }^{\mathrm{a}}$

Robert J. Weyant, DDS, PhD ${ }^{\mathrm{c}}$

Peter F. Ferson, MD ${ }^{\mathrm{b}}$
Introduction: Opinions differ regarding differences between totally musclesparing thoracotomy and standard lateral thoracotomy approaches to pulmonary resection with respect to operative time, postoperative pain and morbidity, and occurrence of chronic postthoracotomy pain syndromes and subjective shoulder dysfunction. Methods: Three hundred thirty-five consecutive patients undergoing muscle-sparing thoracotomy $(n=148)$ or lateral thoracotomy $(n=187)$ to accomplish lobectomy for stage I lung cancer during a 40-month period were evaluated. Local rib resection was not employed, and two chest tubes were routinely used after operation in both thoracotomy groups. Epidural analgesia use was similar after operation in the two groups (muscle-sparing thoracotomy $38 \%$, lateral thoracotomy 38\%). The postoperative hospital courses and patient functional statuses at 1 year were examined. Results: Demographic analyses demonstrated no differences between groups in age, sex, or association of significant comorbid medical illness. Although the operative time required for muscle-sparing thoracotomy was shorter, there were no differences between thoracotomy approaches in any of the other primary acute postoperative variables analyzed (chest tube duration, length of hospital stay, postoperative narcotic requirements, and postoperative mortality). The frequencies of chronic pain and shoulder dysfunction assessed 1 year after operation were also similar between thoracotomy groups. Conclusions: The relative efficacies and rates of occurrence of acute or chronic morbidity are equivalent after muscle-sparing thoracotomy and standard lateral thoracotomy. Although muscle-sparing thoracotomy may possibly be performed more expediently, it appears that the singular advantage of muscle-sparing thoracotomy over standard lateral thoracotomy involves the preservation of chest wall musculature in case rotational muscle flaps should be needed later. (J Thorac Cardiovasc Surg 1996;112:1346-51)
From the Divisions of Cardiothoracic Surgery, Allegheny General Hospital Campus, allegheny University of the Health Sciences, University of Pittsburgh, ${ }^{\mathrm{b}}$ and the Department of Dentistry and Epidemiology, ${ }^{\circ}$ University of Pittsburgh, Pittsburgh, $\mathrm{Pa}$.

Read at the Seventy-sixth Annual Meeting of The American Association for Thoracic Surgery, San Diego, Calif., April 28-May 1, 1996.

Received for publication May 6, 1996; revisions requested May 24, 1996; revisions received July 25, 1996; accepted for publication July 29, 1996.

Address for reprints: Rodney J. Landreneau, MD, Section Head, General Thoracic Surgery, Allegheny General Hospital, Suite 302, 490 E. North Ave., Pittsburgh, PA 15212.

Copyright (C) 1996 by Mosby-Year Book, Inc.

$0022-5223 / 96 \$ 5.00+0 \quad \mathbf{1 2 / 6 / 7 6 9 1 9}$ onsiderable discussion persists regarding the rel$\checkmark$ ative merits of muscle-sparing thoracotomy versus standard lateral thoracotomy as approaches to uncomplicated pulmonary lobar resection. Advocates of muscle-sparing approaches claim that acute and late postoperative pain and morbidity are reduced when these approaches are chosen in preference to standard lateral thoracotomy, in which the primary lateral chest wall muscles (latissimus dorsi and serratus anterior) are severed during entry into the chest. ${ }^{1-9}$

Surgeons who favor a latissimus dorsi-severing approach discount these stated differences. They also argue that the use of modern postoperative pain control measures means that little merit can be found in applying these more "time-consuming" and technically demanding thoracotomy approaches. 
The surgeons of our group were equally divided with respect to these thoracotomy approach concepts. Two surgeons exclusively used muscle-sparing thoracotomy to accomplish uncomplicated pulmonary resection; the other two used standard lateral thoracotomy. The closely balanced clinical experience of our surgeons allowed us to prospectively investigate the relative utility of these thoracotomy approaches for pulmonary lobectomy.

\section{Materials and methods}

During a 40-month period, 335 consecutive patients undergoing lobectomy at our institution for stage I nonsmall-cell lung cancer were operated on with either a muscle-sparing or a standard lateral thoracotomy. The choice of thoracotomy approach was based completely on the individual surgeons' clinical impressions of the utility of their preferred thoracotomy access. During the period of evaluation, muscle-sparing thoracotomy was used in 148 patients undergoing lobectomy and standard lateral thoracotomy was used in 187 of these patients. Patients requiring pneumonectomy or chest wall resection with the lobectomy procedure were excluded from this investigation. The mean values of age, sex, and frequency of comorbid disease were similar between these patient groups.

Although several forms of muscle-sparing thoracotomy have been described in the literature ${ }^{1-9}$ we will describe in detail the techniques of the axillary thoracotomy and lateral muscle-sparing thoracotomy used by us. ${ }^{2,9}$ The axillary approach was used for upper-lobe resection and the lateral approach was used for middle-lobe and lowerlobe resections. The surgeons of our group relying on the standard lateral thoracotomy approach used it for all of their lobar resections. This approach will also be described. Selective lung ventilation to allow ipsilateral lung collapse was achieved in all patients with the aid of a double-lumen endotracheal tube or bronchus blocker technique.

Technique of axillary muscle-sparing thoracotomy. As stated, the axillary muscle-sparing thoracotomy was chosen for resection of upper-lobe lesions. The patient is positioned in a lateral decubitus position with a slight posterior tilt. The surgeon stands on the forward side of the patient. A $10 \mathrm{~cm}$ incision a few centimeters below the axillary hair line, beginning at the lateral aspect of the pectoralis major, is extended posteriorly 1 or $2 \mathrm{~cm}$ beyond the border of the latissimus dorsi. The incision is carried down to the facia of the serratus anterior muscle. This incision routinely gains access to the third intercostal space. While traversing the axillary subcutaneous tissues, it is important for the surgeon to be aware of the intercostobrachial vascular bundle, which requires division to enter the third interspace. The anterior muscle slips of the serratus anterior over the third and fourth ribs are then encountered. These are separated over the interspace to expose the underlying intercostal muscle. A small Richardson retractor is placed beneath the belly of the serratus anterior to further expose the posterior extension of the interspace. The intercostal muscle is then separated from the dorsal aspect of the fourth rib with the use of the electrocautery. The pleura is incised and the intercostal incision is then extended posteriorly from $2 \mathrm{~cm}$ lateral to the mammary arterial pedicle to the paraspinous musculature. Rib resection is not performed. A small Finochietto retractor is positioned within the interspace to widen the intercostal opening. A second Finochietto retractor is then positioned in a right-angled overlapping fashion with respect to the first retractor to displace the serratus anterior and latissimus dorsi posteriorly and the pectoralis major anteriorly., 4, 5,9 This axillary musclesparing approach gives exceptional access to the pulmonary hilum but limited exposure of the middle and lower lobes. After upper lobectomy, two $28 \mathrm{~F}$ chest tubes are positioned at the apex of the chest through separate lower intercostal access sites. Three paracostal sutures are used to stabilize the interspace. The wound is then closed with running absorbable suture. If epidural analgesia has not been accomplished, $2 \mathrm{ml} 0.25 \%$ bupivocaine is infiltrated in a subpleural location about the intercostal neurovascular bundle from the first through the fifth interspace before placement of the paracostal sutures. ${ }^{10,11}$

Technique of lateral muscle-sparing thoracotomy. The lateral muscle-sparing thoracotomy approach begins with placing the patient in a "true" lateral decubitus position. A transverse 10 to $12 \mathrm{~cm}$ incision is performed from the anterior axillary line to the midportion of the belly of the latissimus dorsi muscle, approximately over the fifth or sixth interspace. Subcutaneous flaps are established over the body of the latissimus dorsi from the scapular tip to the level of the ninth rib., ${ }^{4,5} 9$ This subcutaneous dissection is also advanced anteriorly over the serratus anterior muscle slips from the fourth to the eighth intercostal spaces. This subcutaneous dissection is required to accomplish retraction of the latissimus dorsi and serratus anterior musculature for optimal thoracotomy exposure. A Richardson retractor is positioned beneath the medial aspect of the latissimus dorsi to retract it posteriorly for exposure of the lateral aspect of the serratus anterior muscle belly. The posterior fascial membrane of the serratus anterior is then incised along the posterior length of the muscle to gain access to the rib cage. Richardson retractors are used to elevate and displace the belly of the serratus anterior medially to expose the fifth or sixth interspace. A fifth interspace access is used to accomplish middle-lobe resection and a fifth or sixth interspace entry is used for lower-lobe resection. The medial and lateral extents of the intercostal incision are similar to those of the axillary thoracotomy approach, as is the use of dual Finochietto retractors to accomplish rib spreading and displacement of the lateral chest wall musculature. Chest closure is also similar to that in the axillary muscle-sparing approach, but a right-angled $28 \mathrm{~F}$ chest tube is used to drain the posterior costophrenic recess after lower lobectomy and $10 \mathrm{~mm}$ Jackson-Pratt closed suction devices are positioned beneath the subcutaneous flaps to avoid the complication of postoperative seroma formation. $4,5,9$ Bupivocaine intercostal nerve blocks are performed for similar circumstances as in axillary muscle-sparing thoracotomy.

Technique of standard lateral thoracotomy. The skin incision used for the standard lateral thoracotomy is the 
Table I. Postoperative parameters assessed

\begin{tabular}{|c|c|c|c|}
\hline & Muscle-sparing & Standard lateral & $p$ \\
\hline Operative time (min) & $161 \pm 73$ & $198 \pm 82$ & $0.0006^{*}$ \\
\hline Chest tube days & $7.3 \pm 4$ & $6.5 \pm 4$ & 0.18 \\
\hline Major morbidity (\%) & 19 & 21 & 0.61 \\
\hline Hospital stay (days) & $12.4 \pm 12$ & $12.4 \pm 12$ & 0.97 \\
\hline $\begin{array}{l}\text { Postop morphine } \\
\text { (mg/hospital-day) }\end{array}$ & 15.2 & 18.1 & 0.45 \\
\hline Chronic pain (\%) & 1. & 7 & 0.80 \\
\hline $\begin{array}{l}\text { Should function at } 1 \mathrm{yr} \\
\text { (\% preoperative) }\end{array}$ & 97 & 92 & 0.07 \\
\hline Postoperative mortality & $5 / 148(3 \%)$ & $5 / 187(2.6 \%)$ & 0.71 \\
\hline
\end{tabular}

*Significant difference.

same as that used for lateral muscle-sparing thoracotomy. The unique feature of this approach is the division of the belly of the latissimus dorsi in its midportion. This exposes the posterior aspects of the serratus anterior muscle at its junction with the fascia connecting it to the paraspinous musculature. This fascia is incised as in the lateral musclesparing thoracotomy approach to gain access to the intercostal spaces below. The lower aspect of the serratus anterior is then separated from its intercostal attachments to expose the intercostal space to be opened. As in the two muscle-sparing thoracotomy approaches used, no rib resection is performed. A single medium Finochietto retractor is used for rib spreading to gain access for the pulmonary resection. The postresectional aspects of this operative approach are similar to those of the musclesparing thoracotomies described.

Postoperative care and clinical evaluation. The postoperative management was uniform for all patients because they were admitted to a specialized unit of general thoracic surgical patients with common, defined care practices. Epidural catheters were routinely removed by the fourth postoperative day. When this epidural analgesic support was not used, patient-controlled analgesia with intravenous morphine was used. Conversion to patientcontrolled analgesia was also used for all patients after termination of epidural analgesia until the thoracotomy pain could be managed with oral agents. This pain control management was primarily under the direction of our anesthesia pain service and provided us with an independent assessment of the degree of patient pain after the various thoracotomy approaches. The effectiveness of pain control management, as determined by the amount of narcotic analgesia ordered by these independent physicians, was investigated in this study. The postoperative in-hospital courses of these patients were also analyzed with respect to chest tube duration, occurrence of complications, and length of hospital stay.

The postoperative disability and pain of the patients at the 1-month point was not assessed in this study because information regarding this point has been discussed by others., ${ }^{9} 12$ We chose instead to assess the chronic disability at the 1-year mark after the muscle-sparing and standard lateral thoracotomy approaches. At this 1-year point of follow-up, most patients were seen in our clinic and a visual-analog scale assessment of postthoracotomyrelated pain was obtained. ${ }^{13,14} \mathrm{~A}$ visual-analog scale value
Table II. Postoperative complications

\begin{tabular}{lcc}
\hline & Muscle-sparing & Standard lateral \\
\hline Total & $28 / 148(19 \%)$ & $39 / 187(21 \%)^{*}$ \\
Pneumonia & 4 & 8 \\
Empyema with peripheral & 2 & 1 \\
$\quad$ bronchopleural fistula & & \\
Reexploration for bleeding & 0 & 1 \\
Cardiac arrhythmias & 11 & 16 \\
Myocardial infarction & & 13 \\
Prolonged airleak (>7 days) & 8 & 0 \\
Seroma & 3 & \\
\hline
\end{tabular}

${ }^{*} p=0.61$ (total complications).

of 3 or greater was considered a positive indication of late postthoracotomy discomfort. An inquiry into the subjective sense of shoulder girdle function was also performed, with a $0 \%$ to $100 \%$ scale defining the perceived level of strength in the ipsilateral upper extremity compared with the side not operated on.

Statistical analysis of difference in postoperative variables was accomplished by means of life-table analysis obtained by Wilcoxon rank-sum test.

\section{Results}

Although the operative time required for the muscle-sparing thoracotomy approach group was less, our analyses demonstrated no differences in postoperative chest tube duration, narcotic requirements, or hospital stay (Table I). Although the relative occurrence of intraoperative rib fracture between thoracotomy approaches was not quantitated during this period of study, all of us considered this to be an uncommon happening in general. The frequency and nature of postoperative complications were similar between thoracotomy approaches, except for the unique development of subcutaneous seromas requiring aspiration in three patients undergoing the lateral muscle-sparing thoracotomy approach (Table II). The frequencies of chronic pain and shoulder dysfunction assessed 1 year after operation were also similar between thoracotomy groups (Table I). Finally, results of standard pulmonary spirometry at the 1-year postoperative mark were equivalent between muscle-sparing and standard lateral thoracotomy groups.

In both of the cases of postresectional empyema with bronchopleural fistula after muscle-sparing thoracotomy, rotation of the latissimus dorsi muscle into the chest to obliterate the space quickly controlled the problem. ${ }^{15,16}$ Empyema tube drainage was used for several weeks before reexploration and rotation of remnants of serratus anterior, latissimus dorsi, and pectoralis major muscles into the pleural space for the one patient with a postoperative space after standard lateral thoracotomy. In this circum- 
stance, the space was difficult to fill completely with the muscle remnants, and thoracoplasty with the upper three ribs was also required.

\section{Comments}

Muscle-sparing thoracotomy has been advocated as a means of reducing the perioperative morbidity associated with muscle-severing lateral thoracotomy approaches. Such muscle-sparing thoracotomy approaches are aimed at reducing excessive chest wall trauma and preserving shoulder girdle function. These muscle-conserving incisions have also been proposed as measures that can improve early postoperative pulmonary function and reduce the occurrence of complications. It is important, however, to reiterate that most of the reports advocating musclesparing thoracotomy in the literature compare the differences in morbidity between these approaches and the "classic" posterolateral thoracotomy. ${ }^{1-9}$ The posterolateral thoracotomy approach is characterized by a large incision, often extending from the anterior axillary line to well posterior to the scapula. Complete division of the major chest wall musculature (latissimus dorsi and serratus anterior) and paraspinous fascia is routine. The rib at the intercostal entry site is often resected, and wide spreading of the ribs is performed. Trauma to the costovertebral joints posteriorly and to the anterior costal cartilage attachments can be significant. The collective injury to the chest wall can result in significant postoperative pain, leading to respiratory complications. Prolonged shoulder dysfunction and chronic postthoracotomy pain syndromes are common.

The standard lateral thoracotomy approach used by many surgeons today is a significantly less extensive modification of the "classic" posterolateral thoracotomy previously required to approach intrathoracic pathologic processes. The partial sparing of the serratus anterior muscle, avoidance of rib resection, and avoidance of excessive rib spreading separate the current standard lateral thoracotomy technique from the classic posterolateral thoracotomy incision. This reduction in chest wall "skeletal" trauma, rather than the avoidance of division of the latissimus dorsi, is believed by advocates of standard lateral thoracotomy to be the primary determinant in reducing postoperative pain. Because both the standard lateral and muscle-sparing thoracotomy approaches described here focus similarly on reducing such trauma to the chest cage, it is not surprising that there was little difference between approaches in acute postoperative morbidity, in-hospital nar- cotic requirements, and occurrence of chronic postthoracotomy pain syndromes. Although Hazelrigg and colleagues ${ }^{9}$ demonstrated objective differences in early postoperative shoulder function favoring muscle-sparing thoracotomy over standard lateral approaches, these functional differences were no longer apparent by the 1-month postoperative visit. Our investigation also demonstrated no late differences in shoulder function between thoracotomy approaches.

It is important to remember that the ability to accomplish pulmonary resection through less extensive thoracotomy openings is largely a consequence of advances in effective single-lung ventilatory techniques allowing effective ipsilateral lung collapse during the course of lobectomy. Similarly, advances in postoperative pain control management have reduced the incisional pain associated with thoracotomy. Universal use of postoperative epidural catheter analgesia is now our practice during the first 3 to 4 postoperative days; however, this anesthesia support was not available in the earlier part of this investigation. Some reduction in early postoperative pain may be achieved with muscle-sparing techniques, but the use of postoperative epidural pain management may make these differences a moot issue. This use of epidural analgesia has also significantly reduced the acute pulmonary disability in our patients, which may actually counter any minor benefits in early postoperative pulmonary function achieved with muscle-sparing approaches. ${ }^{1,9}$

Although we found the operative time to be shorter with the muscle-sparing thoracotomy approaches, there were no other important differences in the postoperative courses of patients undergoing either muscle-sparing or standard lateral thoracotomy. Pleural space problems associated with empyema or bronchopleural fistula were equally uncommon after either thoracotomy approach. In these rare instances, however, the availability of an intact latissimus dorsi and serratus anterior muscle assisted in management of these problems. Nevertheless, the proximal remnants of these muscles and the pectoralis musculature combined with thoracoplasty can usually be used to manage such space problems in patients undergoing standard lateral thoracotomy. ${ }^{15,16} \mathrm{We}$ therefore conclude that beyond the aesthetic aspects of preserving rather than severing the large lateral chest wall musculature during the course of muscle-sparing thoracotomy, the only potential 
advantages of these approaches over standard lateral thoracotomy involve the possible reduced operative time required and the availability of the these muscles for rotational flap control of postresectional space problems related to bronchopleural fistula or postresectional empyema.

\section{REFERENCES}

1. Lemmer JH, Gomez MN, Symreng T, Ross AR, Rossi NP. Limited lateral thoracotomy: improved postoperative pulmonary function. Arch Surg 1990;125:873-8.

2. Fry WA, Kehoe TJ, McGee JP. Axillary thoracotomy. Am Surg 1990;56:40-4.

3. Horowitz MD, Ancalmo N, Oschner JL. Thoracotomy through the auscultory triangle. Ann Thorac Surg 1989;47: 782-3.

4. Bethencourt DM, Holmes EC. Muscle sparing posterolateral thoracotomy. Ann Thorac Surg 1988;45:337-42.

5. Mitchell RL. The lateral limited thoracotomy incision: standard for pulmonary operation. J Thorac Cardiovasc Surg 1990;99:590-6.

6. Pecora DV. A functional and cosmetically superior thoracotomy incision. Surg Gynecol Obstet 1957;104:626-8.

7. Noirclerc M, Dor V, Chauvin G, et al. La thoracotomie laterale large sans section musculaire. Ann Chir Thorac Cardiovasc 1973;12:181-4.

8. Baeza OR, Foster ED. Vertical axillary thoracotomy: a functional and cosmetically appealing incision. Ann Thorac Surg 1988;123:113-4.

9. Hazelrigg SR, Landreneau RJ, Boley TM, Priestmeyer ML, Schmaltz RA, Nawarawong W, et al. The effect of musclesparing versus posterolateral thoracotomy on pulmonary function, muscle strength and postoperative pain. J Thorac Cardiovasc Surg 1991;101:394-401.

10. Jayr C, Mollie A, Bourgain JL, et al. Postoperative pulmonary complications: general anesthesia with postoperative parenteral morphine compound with epidural analgesia. Surgery 1988;104:57-63.

11. Asantilla R, Rosenberg PH, Scheinin B. Comparison of different methods of postoperative analgesia after thoracotomy. Acta Anaesth Scand 1986;30:421-5.

12. Reading AE. Testing pain mechanisms in persons in pain. In: Wall PD, Melzack R, editors. Textbook of pain. New York: Churchill Livingstone, 1984:196-7.

13. Landreneau RJ, Hazelrigg SR, Mack MJ, Perrino MK, Nunchuck S, Ritter PS, et al. Postoperative pain-related morbidity: video-assisted thoracic surgery vs thoracotomy. Ann Thorac Surg 1993;56:1285-9.

14. Landreneau RJ, Mack MJ, Hazelrigg SR, Naunheim K, Dowling RD, Magee MJ, et al. Prevalence of chronic pain following pulmonary resection by thoracotomy or videoassisted thoracic surgery. J Thorac Cardiovasc Surg 1994;107: 1079-86.

15. Arnold PG, Pairolero PC. Chest wall reconstruction: Experience with 1100 consecutive patients. Ann Surg 1984;199: 725-9.

16. Anderson TM, Miller JI. Use of pleura, azygos vein, pericardium and muscle flaps in tracheo-bronchial surgery. Ann Thorac Surg 1995;60:729-33.

\section{Discussion}

Dr. Douglas J. Mathisen (Boston, Mass.). I congratulate you on a careful analysis of the one thing that all of our patients have in common, their incision. In an era when shorter hospital stays are the norm, we need to pay close attention to those issues that affect length of stay and patient comfort. I think that your study will refocus our attention on trying to improve the way in which we perform incisions and on closer attention to the issues of pain and late-term function.

I have often wondered whether, when we do true muscle-sparing incisions and retract the muscles, we cause injury to the nerves that would affect muscle function. The most obvious example of this would be injury to the long thoracic nerve, with resulting winged scapula. Did you assess muscle function in your early and late follow-up? Were there any cases in which the muscle-sparing incision was abandoned and converted to a thoracotomy? If so, what particular problems did it cause you when you had to do that? You alluded to the one difference being length of operative time. Do you have any sense of how much opening and closing the incision contributed to that difference? Obviously, it is a relatively small percentage of the overall length of any operation, but since you made the point I was wondering whether you had an opportunity to look at that to see if there really is a major difference in the time between the two incisions.

Dr. Landreneau. The first inquiry regarded the incidence of wing scapula after axillary muscle-sparing thoracotomy. We did not specifically look at this entity. In retrospect, however, I do remember two patients who did have a winged scapula after the axillary-thoracotomy approach. What were the other two questions?

Dr. Mathisen. Were there any cases in which you abandoned the axillary muscle-sparing incision?

Dr. Landreneau. No, there were none. I think that with adequate subcutaneous tissue mobilization, as described in Willard Fry's earlier report, and the development of experience with the hilar dissection through this access can allow successful upper-lobe resection or pneumonectomy in most circumstances. This approach is, however, inadequate to approach lower-lobe resection.

Dr. Mathisen. Do you have some sense of how much the actual incision contributed to the decrease in operative time? Obviously if you have got a tougher lobectomy through a bigger incision, the length of time could be quite different.

Dr. Landreneau. We specifically looked at our stage I peripheral lesions to overcome the problems of postoperative recurrence, local recurrence affecting the postoperative pain, and the ability to accomplish the procedure through these less-extensive muscle-sparing techniques. I therefore believe that these techniques did not affect the overall time with respect to the study population. Steve Hazelrigg, in his article of 1991, pointed out that entry into the chest takes longer with the lateral muscle-sparing technique but closure is significantly abbreviated. This resulted in equivalent operative time between musclesparing and muscle-severing approaches in his series.

Dr. Joe B. Putnam, Jr. (Houston, Tex.). At the University of Texas M.D. Anderson Cancer Center, the surgeons are also equally divided regarding whether muscle-sparing 
or standard muscle-cutting approaches should be used. One advantage is that multiple operations and access to the chest might be somewhat easier, particularly in patients who may potentially require multiple resections for pulmonary metastases or second operations for recurrent lung cancer. In addition, the preservation of the chest wall musculature after the operation may enhance recovery of the ipsilateral upper extremity.

Dr. Victor F. Trastek (Rochester, Minn.). A couple of quick questions. Pain certainly remains a mystery after any chest operation, whether thoracoscopic or incisional. I think we have all come to appreciate that. What procedures were performed? Were you limited at all with respect to what procedures you could perform through the limited incision or limited muscle-sparing incision? I did not notice that you told us what operation, lobectomy or pneumonectomy. Second, do you have any idea how many people ended up with rib fractures despite not cutting the rib, in whom once the chest was opened the ribs were fractured along the way. I think that happens from time to time. Third, what are you doing currently, how do you operate on the patient today now that you have learned what you have learned?

Dr. Landreneau. The incidence of rib fracture was not tabulated in this study. I know that rib fracture occurred in a minority of patients in both groups, but I do not have this information at hand.
With adequate mobilization of subcutaneous flaps, muscular retraction can be readily accomplished. With careful, slow opening of the chest, as we all know, you will avoid the problem of rib fracture in most instances with both muscle-sparing and muscle-severing thoracotomy approaches. The axillary approach is really an exceptional approach to upper-lobe resection; that is what we limited it to, and we had no difficulty with that. When we had a middle-lobe or lower-lobe resection, we relied on a muscle-sparing or standard lateral thoracotomy approach.

With respect to what we are going to do in the future, I think that the surgeons participating in this study were equally bullheaded, and I am not sure that this study is going to change our opinions about our approaches to thoracotomy. I am not going to break the code regarding who did which incision at this point. At this point, there appears to be little objective difference between musclesparing and muscle-severing approaches to enter the chest with respect to postoperative pain and morbidity as long as careful and thoughtful techniques are used. With the advent of epidural analgesia, I think that the minimal short-term perioperative benefits that may be seen with muscle-sparing techniques are obviated. I conclude by saying again that the major advantage of muscle-sparing thoracotomy techniques is the preservation of chest wall musculature in case local muscle flaps should be needed after operation. 